\title{
First principles study of barium chalcogenides
}

\author{
Gökhan Gökoğlu ${ }^{\mathrm{a}, \mathrm{b}, *}$ \\ a Department of Physics, Karabük University, 78100 Karabük, Turkey \\ ${ }^{\mathrm{b}}$ Department of Physics, Bilkent University, 06800 Bilkent, Ankara, Turkey
}

\section{A R T I C L E I N F O}

\section{Article history:}

Received 21 June 2008

Accepted 14 August 2008

\section{PACS:}

62.20.de

62.20.dj

63.20.dk

Keywords:

A. Chalcogenides

C. Ab initio calculations

D. Elastic properties

D. Phonons

\begin{abstract}
A B S T R A C T
In this study, ab initio calculation results of the vibrational properties and elastic parameters as well as characteristic Debye temperature and Poisson's ratios of two barium chalcogenides, BaSe and BaS, which crystallize in NaCl-type structure, were presented. Calculations were based on plane wave basis sets together with ultrasoft pseudopotentials in the framework of density functional theory (DFT) with generalized gradient approximation. Phonon dispersion spectra were obtained using the first principles linear response approach of the density functional perturbation theory (DFPT). The detailed total energy calculations were performed in order to obtain elastic constants using distortions on cubic phase. The calculated structural, elastic, and thermal parameters of BaSe and BaS systems agree well with the available experimental data and theoretical calculations.
\end{abstract}

(c) 2008 Elsevier Ltd. All rights reserved.

\section{Introduction}

The alkaline-earth chalcogenides, which are the compounds of II-VI group elements, are large gap insulators with closed shell ionic system and most of them crystallize in six-fold coordinated $\mathrm{NaCl}$-type structure except MgTe whose stable ground state phase is hexagonal wurtzite structure under zero pressure. It is also known that the barium chalcogenides have the highest ionic character among the all other alkaline-earth chalcogenide compounds and show metallization behavior [1,2] together with a structural phase transition under high pressures [3]. The importance of these materials arise from the large technological applications in microelectronics, light-emitting diodes (LEDs), laser diodes (LDs), and magneto-optical devices [4].

The electronic structure and optical properties of these compounds ( $\mathrm{BaX} ; \mathrm{X}=\mathrm{Se}, \mathrm{S}, \mathrm{Te}$ ) were investigated in previous experimental [5,6] and theoretical studies [3,7-10]. The phase transition behavior from $\mathrm{NaCl}(B 1)$ to $\mathrm{CsCl}(B 2)$ structures were also studied extensively. In these works, the stability of $B 1$ phase were verified up to $\sim 6 \mathrm{GPa}$ for $\mathrm{BaS}$ and BaSe compounds $[1,11]$. The band-overlap metallization and metal-insulator transition volumes of barium chalcogenides were calculated for the first

\footnotetext{
* Corresponding author at: Department of Physics, Bilkent University, 06800 Bilkent, Ankara, Turkey. Tel.: +90312 2902151 ; fax: +903122664579.

E-mail address: ggokoglu@fen.bilkent.edu.tr
}

time in an earlier work using self-consistent augmented spherical wave (ASW) technique with local density approximation [12]. In two recent studies, electronic, high pressure, and elastic properties as well as chemical bonding mechanisms of BaTe, BaSe, and BaS were investigated by FP-LAPW method with generalized gradient approximation (GGA) functionals [7,13]. Electronic and optical properties were also investigated in another work using the same method with and without spin-orbit interactions [10]. It is a noteworthy point that spin-orbit interactions have no remarkable effect on electronic band structures of these compounds. Although the electronic properties of related compounds are understood well, the studies on phonon and elastic properties are very rare.

In the present work, elastic, structural, and vibrational properties of BaSe and BaS systems in stable ground state phase (B1 structure) were studied using plane-wave pseudopotential method of the density functional theory (DFT) with GGA functionals. The vibrational properties were investigated in the linear response approach of the density functional perturbation theory (DFPT) in which phonon frequencies can be obtained with an accuracy level which is equivalent to that previously obtained for electronic structure. To date, there are only a few studies in literature about the elastic properties of these compounds and no study on vibrational dynamics, although there is a direct relation between elastic and phonon properties. Moreover, several fundamental physical properties of solid materials like specific heat and thermal expansion are related to elastic and lattice dynamic 
properties. Therefore, the major goal of the present study is to clarify the lattice dynamics and all elastic and thermal parameters of related compounds due to the absence of convincing studies in the literature.

\section{Computational details}

Exchange-correlation potential was approximated with GGA functionals of the DFT with Perdew-Burke-Ernzerhof parameterization [14]. GGA is more successful to describe the ground state properties of several binary compounds and experimental findings can be verified with high accuracy. Pseudopotentials are generated by scalar relativistic calculation for Ba and S and nonrelativistic calculation for Se atoms with non-linear core correction. The valance states of the atoms considered are as follows; Ba: $6 s^{2}$, S: $3 s^{2} 3 p^{4}$, Se: $4 s^{2} 4 p^{4}$. Brillouin zone integration was performed with automatically generated $10 \times 10 \times 10$ special k-point mesh following the convention of Monkhorst and Pack [15]. Wave-functions were expanded in plane wave basis sets up to a kinetic energy cut-off value of $950 \mathrm{eV}$. These k-point and energy cut-off values were tested and provide convergence in selfconsistent calculations. The charge density cut-off value was taken as 10 times of the kinetic energy cut-off value due to the high ionic character of the compounds under study. It is known that the low charge density cut-off values can cause convergence problems for these kinds of materials. Spin-orbit interactions were not included in calculation procedure.

Phonon dispersion spectra was constructed using DFPT in the linear response approach [16-18], in which second order derivatives of the total energy were calculated to obtain dynamical matrix. This method enables one to calculate the vibrational properties of materials with high level of accuracy. A relatively higher kinetic energy cut-off value with $1220 \mathrm{eV}$ was used for phonon calculation in order to obtain better accuracy. The energy threshold value for convergence was $1.0 \times 10^{-14} \mathrm{Ry}$. Dynamical matrices were calculated in a uniform grid of $4 \times 4 \times$ $4 \mathrm{k}$-points in the irreducible part of the Brillouin zone. The full phonon dispersion spectra can be obtained with Fourier transform using dynamical matrices.

The bulk moduli and its pressure derivatives are calculated with Vinet equation of states [19] using energy-volume variation. The root mean square error of energy obtained in fitting process was less than $1.0 \times 10^{-6}$ Ry indicating high accuracy.

There are three independent elastic constants in cubic crystals: $C_{11}, C_{12}$, and $C_{44}$. These coefficients were calculated using volume conserving isochoric strains, which cause tetragonal and orthorhombic distortions on cubic structure. All the details of elastic constant calculation procedure including strain tensors used were explained in detail in Refs. [20,21].

\section{Results and discussion}

\subsection{Elasticity of BaS and BaSe}

The elastic parameters of the related compounds were calculated using tetragonal and orthorhombic distortions on cubic NaCl-type (space group $F m \overline{3} m$ ) structures. The utilization of isochoric (volume conserving) strains in elastic constants calculations has several important consequences [22]. The total energy of the system depends on volume more strongly than on strain. Therefore, the volume contribution to total energy can be eliminated by using volume conserving strains. The systems are fully relaxed after each distortion in order to reach the equilibrium state with approximately zero forces on all atoms. This calculation procedure for elastic constants is the most efficient way and gives very accurate results in comparison to initial slopes of phonon dispersion spectra. The obtained root mean square deviations for fitting process were less than $0.2 \%$ which is an indication of the accuracy of calculated values.

GGA calculation of all structural and elastic parameters of BaS and BaSe compounds are presented in Table 1 . There is no experimental study in the literature about the elastic constants of barium chalcogenides, hence there is no possibility for comparison with experiments. But the calculated values of lattice constants and bulk moduli are in good agreement with experiments. In calculation of the bulk moduli values, I used Vinet equation of states which is found to be most accurate in a previous comprehensive study [23]. Murnaghan [24] and thirdorder Birch [25] equation of states give approximately $10 \%$ lower bulk moduli values. These values are also consistent with the other theoretical works of elastic constants based on LDA and GGA functionals. The GGA calculated lattice constant values are greater than LDA values due to overestimation. Surprisingly, my calculated $C_{12}$ and $C_{44}$ values are in better agreement with LDA results rather than GGA. The given $C_{12}$ and $C_{44}$ values of the other GGA based theoretical work are too low and do not seem to be reasonable.

It is worth noting that the elastic constants of BaS including bulk modulus are higher than the BaSe compound. This shows that the elastic resistivity of $\mathrm{BaS}$ to a pressure in any direction is

Table 1

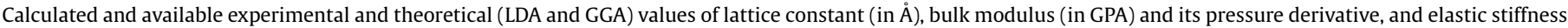
coefficients (in GPa) of BaS and BaSe systems in rocksalt structure

\begin{tabular}{|c|c|c|c|c|}
\hline Parameters & Present & Exp. & Theo. [LDA] (Ref. [13]) & Theo. [GGA] (Ref. [7]) \\
\hline \multicolumn{5}{|l|}{$\mathrm{BaS}$} \\
\hline$a$ & 6.352 & 6.389 (Ref. [32]) & 6.316 & 6.446 \\
\hline$B$ & 44.60 & 39.42 (Ref. [32]) & 53.32 & 40.28 \\
\hline$B^{\prime}$ & 5.15 & & 4.90 & 3.88 \\
\hline$C_{11}$ & 94.57 & & 115 & 101.05 \\
\hline$C_{12}$ & 19.61 & & 17 & 9.67 \\
\hline$C_{44}$ & 18.60 & & 18 & 9.24 \\
\hline \multicolumn{5}{|l|}{ BaSe } \\
\hline$a$ & 6.608 & 6.593 (Ref. [1]) & 6.511 & 6.668 \\
\hline$B$ & 37.21 & 43.4 (Ref. [33]) & 45.95 & 37.05 \\
\hline$B^{\prime}$ & 3.72 & & 4.42 & 3.74 \\
\hline$C_{11}$ & 82.67 & & 104 & 94.74 \\
\hline$C_{12}$ & 14.48 & & 14 & 8.20 \\
\hline$C_{44}$ & 15.62 & & 15 & 8.51 \\
\hline
\end{tabular}


higher than the BaSe structure. The work of Lin et al. [26] states that the ionic character of bonds between barium and other chalcogen atoms decrease with the increasing atomic number of chalcogen atoms. Therefore, the ionic character of the chemical bond in $\mathrm{BaS}$ is stronger than the BaSe resulting in a more stable crystal structure.

The one of the most important property of crystalline solids is elastic anisotropy ratio which is defined as $A=2 C_{44} /\left(C_{11}-C_{12}\right)$. This quantity is calculated as 0.50 and 0.46 for BaS and BaSe, respectively. These crystals cannot be regarded as elastically anisotropic due to the $A$ values much less than 1.0. The larger values of $A$ show the tendency to differ from cubic structure. This situation is also an indication of mechanical stability of the current crystal structures ( $B 1$ phase). Poisson's ratio is a major elasticity related characteristic property for a material and is calculated using the following relation [27]:

$v=\frac{1}{2}\left[\frac{B-(2 / 3) G}{B+(1 / 3) G}\right]$

where $G$ is the isotropic shear modulus in the form $G=\left(G_{V}+G_{R}\right) / 2 . G_{V}$ and $G_{R}$ are Voigt's and Reuss's shear moduli, respectively, and expressed as [28]

$G_{V}=\frac{C_{11}-C_{12}+3 C_{44}}{5}$

$\frac{5}{G_{R}}=\frac{4}{C_{11}-C_{12}}+\frac{3}{C_{44}}$

Debye temperature $\left(\Theta_{D}\right)$ corresponds to the upper limit of phonon frequency in a crystal lattice and calculated using following relation [29]:

$\Theta_{D}=\frac{h}{k_{B}}\left[\frac{3 n}{4 \pi}\left(\frac{N_{A} \rho}{M}\right)\right]^{1 / 3} v_{m}$

where $h$ and $k_{B}$ are universal constants and $n, N_{A}, M$, and $\rho$ are the number of atoms in formula unit, Avogadro's number, molecular mass, and density (calculated values: $\rho_{\mathrm{BaS}}=4.392 \mathrm{~g} / \mathrm{cm}^{3}$, $\rho_{\text {Base }}=4.980 \mathrm{~g} / \mathrm{cm}^{3}$ ), respectively. $v_{m}$ is the average wave velocity and is dependent to transverse $\left(v_{t}\right)$ and longitudinal $\left(v_{l}\right)$ wave velocities due to following formulas [30]:

$v_{m}=\left[\frac{1}{3}\left(\frac{2}{v_{t}^{3}}+\frac{1}{v_{l}^{3}}\right)\right]^{-1 / 3}$

$v_{l}=\sqrt{\frac{3 B+4 G}{3 \rho}}$

and

$v_{t}=\sqrt{\frac{G}{\rho}}$

The calculated values of average, transverse, and longitudinal wave velocities, Debye temperatures, and Poisson's ratios are presented in Table 2. There is no study in the literature about these parameters, hence there is no possibility for comparison. But clearly, the accuracy of these values is as high as elastic constants. The obtained Debye temperatures of these systems are relatively low as an indication of the large contribution of lattice vibrations to specific heat in the low temperature limit.

\section{Table 2}

Calculated values of transverse, longitudinal, average wave velocities, Debye temperatures and Poisson's ratios of BaS and BaSe compounds

\begin{tabular}{llllll}
\hline Structure & $v_{t}(\mathrm{~km} / \mathrm{s})$ & $v_{l}(\mathrm{~km} / \mathrm{s})$ & $v_{m}(\mathrm{~km} / \mathrm{s})$ & $\Theta_{D}(\mathrm{~K})$ & $v$ \\
\hline BaS & 2.37 & 4.20 & 2.64 & 247 & 0.276 \\
BaSe & 2.07 & 3.64 & 2.31 & 208 & 0.258 \\
\hline
\end{tabular}

\subsection{Vibrational properties}

The full phonon dispersion spectra of BaS and BaSe systems along the all main symmetry direction in fcc Brillouin zone are shown in Fig. 1 together with vibrational density of states. The energy scale of acoustic branches is very similar, while there are remarkable differences in optical modes of two compounds due to atomic mass difference of chalcogen atoms. The similarity of initial slopes of the acoustic modes of two compounds under study near $\Gamma$-point is a direct indication of similarity of the elastic constant values which can also be obtained from the slopes of acoustic branches when $\mathbf{q} \Rightarrow 0$. The degenerated two transverse acoustic and optical modes along the $\Gamma-X$ and $\Gamma-L$ directions are splitted along the $\Gamma-K$ direction according to crystal symmetry. There is a visible gap between acoustical and optical modes of BaS due to cation/anion mass ratio. The energy of longitudinal acoustic branch of BaSe along $\Gamma-K$ direction is higher than the lowest transverse optical (TO) mode, this situation can also be seen more clearly in vibrational density of states in which three well-separated regions are distinguished for BaS compound corresponding to transverse and longitudinal acoustical and optical modes. The energies of the longitudinal optical (LO) branches reach their maximum values at $\Gamma$-point for both the compounds. The TO branches of BaS and BaSe do not show dispersive behavior along all the symmetry directions and lie around $\sim 4$ and $\sim 3 \mathrm{THz}$ frequency levels, respectively.

The large splitting of TO and LO modes at the zone center is a characteristic behavior for ionic crystals and the same situation was observed for magnesium chalcogenides in a recent theoretical study [31]. The overall phonon spectra of the barium chalcogenides do not show any anomalous vibrational property as an indication of the stability of the NaCl-type crystal structure.

The elastic parameters of the solids can also be calculated using the initial slopes of phonon dispersion spectra, so the region near $\Gamma$-point are assumed to be linear and the slope gives the wave velocity $v=\mathrm{d} \omega / \mathrm{d} k$. This can be done for several directions, but only the acoustic modes in $\Gamma-K([110])$ direction can give all elastic parameters of a cubic crystal. The three non-degenerated acoustic modes in [110] direction, $T A_{1}, T A_{2}$, and $L A$ correspond to the $C_{44}, C^{\prime}\left(=\left(C_{11}-C_{12}\right) / 2\right)$, and $C_{L}\left(=\left(C_{11}+C_{12}+2 C_{44}\right) / 2\right)$,

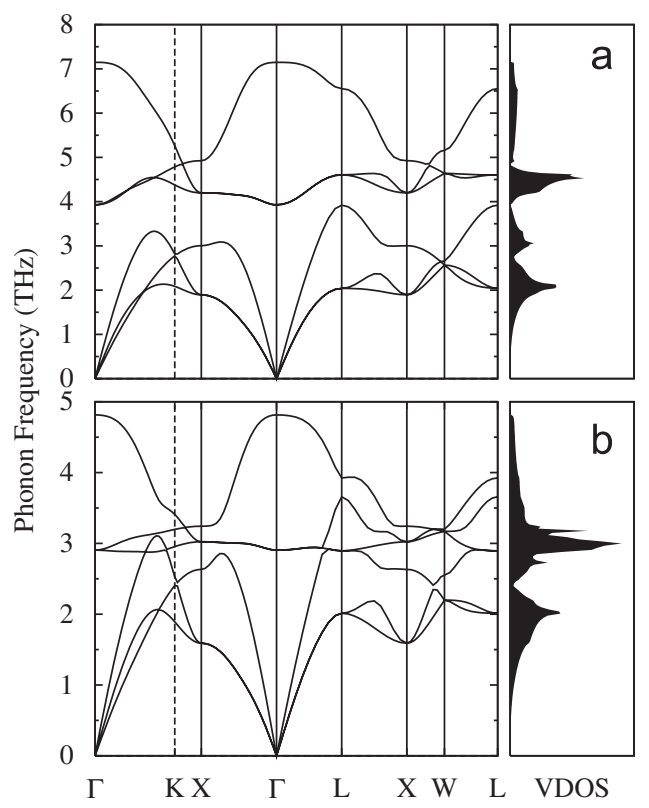

Fig. 1. Phonon dispersion curves along the main symmetry directions in first Brillouin zone and vibrational density of states of BaS (a) and BaSe (b) compounds. 
Table 3

The comparison of elastic parameters calculated using strains and initial slopes $(\mathbf{q} \Rightarrow 0)$ of phonon dispersion curves

\begin{tabular}{lllllll}
\hline Structure & Method & $C^{\prime}$ & $C_{L}$ & $C_{11}$ & $C_{12}$ & $C_{44}$ \\
\hline BaS & Strain & 37.48 & 75.69 & 94.57 & 19.61 & 18.60 \\
& Slope & 32.4 & 73.3 & 84.2 & 19.4 & 21.5 \\
& & & & & & \\
BaSe & Strain & 34.09 & 64.20 & 82.67 & 14.48 & 15.62 \\
& Slope & 29.9 & 63.3 & 75.2 & 15.4 & 18.0 \\
\hline
\end{tabular}

All parameters are in units of GPa.

respectively. The comparison of elastic constants calculated using strain tensors (also given in Table 1) and initial slopes of phonon dispersions are presented in Table 3. Although, the calculation based on distortions are always known to be more accurate, the results of two methods are in very good agreement. This situation is a nice indication of the accuracy of calculated vibrational spectra.

\section{Conclusion}

In this study, elastic and vibrational properties of two highly ionic barium chalcogenide compounds, $\mathrm{BaS}$ and BaSe, were studied within the framework of first principles density functional calculations. The elastic parameters were obtained using volume conserving isochoric strains, which is the most detailed and most accurate way for calculating these constants. Phonon dispersion spectra of related compounds was constructed for the first time using linear response approach. The all calculated elastic and structural parameters are in agreement with the other theoretical works and available experimental data. The fundamental physical properties of crystalline solids like Debye temperature and Poisson's ratio were also calculated for the first time. My all calculated parameters should have high accuracy due to very low root mean square errors in fitting procedures. The calculated phonon spectra does not show any vibrational anomaly, which is also consistent with the low elastic anisotropy ratio.

\section{Acknowledgments}

This research was supported in part by TÜBITAK (The Scientific \& Technological Research Council of Turkey) through TR-Grid e-Infrastructure Project. The author acknowledges TÜBiTAK for research fellowship under Program-2218.

\section{References}

[1] T.A. Grzybowski, A.L. Ruoff, Phys. Rev. B 27 (1983) 6502.

[2] T.A. Grzybowski, A.L. Ruoff, Phys. Rev. Lett. 53 (1984) 489.

[3] G. Kalpana, B. Palanivel, M. Rajagopalan, Phys. Rev. B 50 (1994) 12318.

[4] Y. Nakanishi, T. Ito, Y. Hatanaka, G. Shiomaka, Appl. Surf. Sci. 66 (1992) 515.

[5] S.T. Weir, Y.K. Vohra, A.L. Ruoff, Phys. Rev. B 35 (1987) 874.

[6] A. Jayaraman, B. Batlogg, R.G. Maines, H. Bach, Phys. Rev. B 26 (1982) 3347.

[7] F.E.H. Hassan, H. Akbarzadeh, Comput. Mater. Sci. 38 (2006) 362.

[8] S.H. Wei, H. Kraukauer, Phys. Rev. Lett. 55 (1985) 1200.

[9] H. Akbarzadeh, M. Dadsetani, M. Mehrani, Comput. Mater. Sci. 17 (2000) 81.

[10] A. Pourghazi, M. Dadsetani, Physica B 370 (2005) 35.

[11] S.T. Weir, Y.K. Vohra, A.L. Ruoff, Phys. Rev. B 33 (1986) 4221.

[12] A.E. Carlsson, J.W. Wilkins, Phys. Rev. B 29 (1984) 5836.

[13] A. Bouhemadou, R. Khenata, F. Zegrar, M. Sahnoun, H. Baltache, A.H. Reshak, Comput. Mater. Sci. 38 (2006) 263.

[14] J.P. Perdew, K. Burke, M. Ernzerhof, Phys. Rev. Lett. 77 (1996) 3865.

[15] H.J. Monkhorst, J.D. Pack, Phys. Rev. B 13 (1976) 5188.

[16] X. Gonze, Phys. Rev. A 52 (1995) 1086.

[17] X. Gonze, Phys. Rev. A 52 (1995) 1096.

[18] S. Baroni, S. de Gironcoli, A.D. Corso, Rev. Mod. Phys. 73 (2001) 515.

[19] P. Vinet, J. Ferrante, J.R. Smith, J.H. Rose, J. Phys. C 19 (1986) L467.

[20] O. Gülseren, R.E. Cohen, Phys. Rev. B 65 (2002) 064103.

[21] G. Gökoğlu, A. Erkisi, Solid State Commun. (2008) doi:10.1016/j.ssc.2008. 05.013.

[22] G.S. Neumann, L. Stixrude, R.E. Cohen, Phys. Rev. B 60 (1999) 791.

[23] R.E. Cohen, O. Gülseren, R.J. Hemley, Am. Mineral. 85 (2000) 338.

[24] F.D. Murnaghan, Proc. Natl. Acad. Sci. USA 3 (1944) 244.

[25] F. Birch, J. Geophys. Res. 83 (1978) 1257.

[26] G.Q. Lin, H. Gong, P. Wu, Phys. Rev. B 71 (2005) 085203.

[27] B. Mayer, H. Anton, E. Bott, M. Methfessel, J. Sticht, P.C. Schmidt, Intermetallics 11 (2003) 23.

[28] Y.O. Ciftci, K. Çolakoglu, E. Deligoz, H. Ozisik, Mater. Chem. Phys. 108 (2008) 120.

[29] L. Johnston, G. Keeler, R. Rollins, S. Spicklemire, Solid State Physics Simulations. The Consortium for Upper Level Physics Software, Wiley, New York, 1996.

[30] E. Schreiber, O.L. Anderson, N. Soga, Elastic Constants and Their Measurements, McGraw-Hill, New York, 1973.

[31] S. Duman, S. Bağci, H.M. Tütüncü, G.P. Srivastava, Phys. Rev. B 73 (2006) 205201.

[32] S. Yamaoka, O. Shimomuro, H. Nakazawa, O. Fukunaga, Solid State Commun. 33 (1980) 87.

[33] A.L. Ruoff, T.A. Grzybowski, in: S. Minomura (Ed.), Solid State Physics Under Pressure, Terra Scientific, Tokyo, 1985. 